\title{
Internet of Things in Healthcare
}

\author{
Serik Smagulov, \\ Viktoriya Smagulova MD
}

\begin{abstract}
This article discusses the impact of the Internet of Things (IoT) on the development and modernization of healthcare. The demand for connected devices covers many industries, including healthcare. The Internet of Things revolutionizes patient data collection and records, performs individual health assessments. The author examines various possibilities, recent advances in the field of the Internet of things, including various mobile health monitoring applications, inhalers, smart contact lenses. Special attention is focused on intelligent continuous glucose monitoring (CGM). The progress of the Internet of Things has reached even the launch of smart hospitals. Managers have access to predictive analytics, thanks to which they can solve a problem before it happens. The problem of the help of the Internet of Things when solving problems of chronic diseases is considered. As for the future, the growing interaction of artificial intelligence and the Internet of things in the health sector is likely, according to the author, to create more intelligent IoT devices that can perform actions autonomously. Besides, in the future, such next-generation devices will be able to provide intelligent services, allowing real-time data processing.
\end{abstract}

Key words: Internet of things, healthcare, applications, diabetes, technology, artificial intelligence.

Internet of Things (IoT) is a concept of a computer network of physical objects ("things") equipped with embedded technologies for interacting with each other or with the external environment, considering the organization of such networks as a phenomenon that can restructure economic and social processes, excluding the need for human participation from some actions [1].

Patients, doctors, and medical facilities benefit from the use of IoT in healthcare. And, despite security concerns, the future of IoT in the health sector is bright.

The Internet of things is not new, but recently it has attracted more and more attention. The concept involves the use of electronic devices that collect or track data and connect to a private or public cloud, which allows them to trigger certain events automatically.

The demand for connected devices covers many industries, including healthcare.

Just a few years ago practitioners closely followed the development of IoT to see if this would be part of their future. Today it is not only a reality but also a relief for both medical professionals and patients. 
In the past decade, Internet-connected devices have been presented to patients in various forms. The use of IoT devices has played an essential role in providing more valuable real-time data to doctors and reducing the need for direct patient-doctor interaction [2].

The IoT revolutionizes patient data collection and records, performs individual health assessments [3].

Many applications have appeared to give people more control over their health [4]. For example, sleep apps track sleep cycles to make sure the person gets enough rest. Fitbits track exercises help people stay fit. Calorie planning apps help in cooking; many apps offer an individualized meal plan, so people know what to eat and which food to avoid.

IoT facilitated remote health monitoring system has many benefits over conventional health monitoring system [5]. IoT devices can be used to enable remote health monitoring of patients with chronic diseases such as cardiovascular diseases (CVD) [6], while long-term Electrocardiogram (ECG) monitoring in residential environments has been proposed [7].

Another IoT achievement is Intelligent Continuous Glucose Monitoring (CGM).

Diabetes is fertile ground for the development of intelligent devices since this disease affects about one in ten adults and requires constant monitoring and prescription of treatment.

The Continuous Glucose Monitor (CGM) is a device that helps people with diabetes continuously monitor their blood glucose levels for several days by taking readings regularly. The first CGM system was approved by the US Food and Drug Administration (FDA) in 1999. In recent years, several intelligent CGMs have appeared on the market.

Intelligent CGMs, such as Eversense and Freestyle Libre, send blood glucose data to an app on an iPhone, Android, or Apple Watch allowing the user to check their information and identify trends easily. The FreeStyle LibreLink application also allows for remote monitoring by caregivers.

Another smart device that improves the lives of patients with diabetes is an intelligent pen with insulin. They have the ability to automatically record the time, amount and type of insulin given in a dose, and recommend the correct type of insulin injection at the right time.

The devices interact with a smartphone app that can store long-term data, help diabetics calculate insulin dosage, and even (in the case of Gocap) allow patients to record their 
food intakes and blood sugar levels to see how their diet and insulin consumption affect their levels blood sugar.

IoT also includes connected inhalers. The largest manufacturer of intelligent inhalers is Propeller Health. Instead of producing whole inhalers, Propeller created a sensor that connects to an inhaler or Bluetooth spirometer. It connects to the app and helps people with asthma understand the causes of their symptoms, tracks the use of medications.

One of the advantages of using a connected inhaler is that medications are taken more consistently and more often. The Propeller sensor generates inhaler usage reports that you can share with your doctor and shows if they use it as often as prescribed.

Medical smart contact lenses are another ambitious IoT application in healthcare. Some companies are working on programs for smart lenses which are aimed at the treatment of hyperopia caused by the loss of elasticity of the lens of the eye, and recovery after surgery to remove cataracts. The results, of course, still create skepticism but according to some scientists, this development has the right to exist.

The Swiss company Sensimed has also developed the non-invasive intelligent Triggerfish contact lens which automatically records eye size changes that can lead to glaucoma.

Progress IoT reached even the launch of smart hospitals. Smart Hospital uses related assets to improve services, procedures, and capabilities. Connected assets enhance the functionality of all hospital equipment. The work of maintenance managers will be simplified, as they can remotely control the temperature in the hospital, humidity and air regulation.

Managers will also have access to predictive analytics through which managers can solve a problem before it happens.

IoT reduces emergency waiting time. For example, the Sinai Medical Center in New York effectively cut waiting times for $50 \%$ of emergency room patients who need inpatient care.

In some cases, patients do not even need to visit the emergency room or hospital. One of the most visible and widespread applications of healthcare and IoT is remote health monitoring, sometimes known as telehealth. 
IoT helps with problems of chronic diseases. Utilities such as Fitbit use IoT to monitor personal health. Such information can be passed on to your doctor to help solve recurring problems. A company called Health Net Connect recently created a diabetes care program for the population to improve clinical treatment and reduce the cost of treating patients - and they have already produced some impressive results.

For the future, related technologies can revolutionize the industry and create products such as smart inhalers, smart pills and more, all of which will help people become healthier. Many stakeholders, and especially patients, will benefit from the expansion of IoT in healthcare, so many are keen to invest in this sector.

The Internet of Things (IoT) has opened up a whole world of opportunities in medicine: when connected to the Internet, conventional medical devices can collect invaluable additional data, provide further insights into symptoms and trends, provide remote treatment and generally give patients more control over their lives and treatments [8;9].

Wearable devices have become products that patients now carry, transferring data to doctors and, thus, allowing doctors to track vital signs in real time, including heart rate, glucose level, and even fall detection.

Thanks to medical remote monitoring devices for patients who collect vital data elements in real time, patients can be in the comfort of their homes, while remaining under the close supervision of a medical professional.

In the future, when IoT becomes more common in medical facilities, we will see that next-generation IoT devices provide intelligent services, allowing real-time data processing [10].

This is very important for scenarios in which traditionally devices may require a connection to a server or network. But thanks to the ability to perform processing at the device level, they now do not run the risk of malfunctioning if the device loses connection or is required to work offline. For example, an insulin pump that operates independently of the Internet will continue to analyze glucose levels on the verge — the patient — and release the right amount of insulin and upload data to the cloud when it connects to the Internet. 
How far will IoT go? The growing interaction of artificial intelligence and IoT in the healthcare sector is likely to lead to the creation of more intelligent IoT devices that can perform actions autonomously.

\section{References}

1. J. Höller, V. Tsiatsis, C. Mulligan, S. Karnouskos, S. Avesand, and D. Boyle, From Machine-to-Machine to the Internet of Things: Introduction to a New Age of Intelligence. Amsterdam, The Netherlands: Elsevier, 2014.

2. P. Lopez, D. Fernandez, A. J. Jara, and A. F. Skarmeta, "Survey of Internet of Things technologies for clinical environments,'” in Proc. 27th Int. Conf. Adv. Inf. Netw. Appl. Workshops (WAINA), Mar. 2013, pp. 1349-1354.

3. S. M. Riazul Islam. The Internet of Things for Health Care: A Comprehensive Survey // Digital Object Identifier 10.1109/ACCESS.2015.2437951.

4. W. Zhao, W. Chaowei, and Y. Nakahira, "Medical application on Internet of Things,' in Proc. IET Int. Conf. Commun. Technol. Appl. (ICCTA), Oct. 2011, pp. 660-665.

5. Darshan K. R. and Anandakumar K. R., "A comprehensive review on usage of Internet of Things (IoT) in healthcare system," in Proc. Int. Conf. Emerg. Res. Electron., Comput. Sci. Technol., Mandya, India, Dec. 2015, pp. 132-136.

6. Azariadi D., Tsoutsouras V., Xydis S., and Soudris D., "ECG signal analysis and arrhythmia detection on IoT wearable medical devices," in Proc. 5th Int. Conf. Modern Circuits Syst. Technol., Thessaloniki, Greece, May 2016, pp. 1-4.

7. Spanò E., Di Pascoli S., and Iannaccone G., "Low-power wearable ECG monitoring system for multiple-patient remote monitoring," IEEE Sensors J., vol. 16, no. 13, pp. 5452-5462, Jul. 2016.

8. N. Yang, X. Zhao, and H. Zhang, "A non-contact health monitoring model based on the Internet of Things,', in Proc. 8th Int. Conf. Natural Comput. (ICNC), May 2012, pp. 506-510.

9. M. Diaz, G. Juan, O. Lucas, and A. Ryuga, "Big data on the Internet of Things: An example for the e-health,', in Proc. Int. Conf. Innov. Mobile Internet Services Ubiquitous Comput. (IMIS), Jul. 2012, pp. 898-900. 
10. L. Mainetti, L. Patrono, and A. Vilei, "Evolution of wireless sensor networks towards the Internet of Things: A survey," in Proc. 19th Int. Conf. Softw., Telecommun. Comput. Netw. (SoftCOM), Sep. 2011, pp. 1-6. 